\title{
Identification of Multiple Partial Discharge Sources in High Voltage Transformer Windings
}

\author{
N H Nik Ali ${ }^{1 *}$, J. A. Hunter ${ }^{1}$, P Rapisarda ${ }^{2}$, P L Lewin ${ }^{1}$ \\ ${ }^{1}$ The Tony Davies High Voltage Laboratory, University of Southampton, SO17 1BJ, UK. \\ ${ }^{2}$ Communications, Signal Processing and Control, University of Southampton, SO17 1BJ, UK. \\ *E-mail:nhna1g13@soton.ac.uk
}

\begin{abstract}
Partial discharge (PD) analysis is an important tool for assessing the lifespan of power equipment especially in cables and high voltage (HV) transformers. Different PD sources have different effects on the condition and performance of power equipment insulation system. Therefore, the ability to identify different PD sources is a great interest for both system utilities and equipment manufacturers. Hence, an experiment has been designed at the Tony Davies High Voltage Laboratory, University of Southampton to access the methodologies for the identification of multiple PD sources within a HV transformer windings. Previous work at Southampton developed a non-linear based technique that facilitates identification of the location of a PD source within an interleaved winding. This project is concerned with the feasibility of locating several sources simultaneously based only on measurement data from wideband RFCTs placed at the neutral to earth point and the bushing tap-point to earth. Initial results from a simple experiment are presented and development of an analytical approach described.
\end{abstract}

\section{INTRODUCTION}

Condition monitoring of electrical equipment, such as $\mathrm{HV}$ power transformers, benefits both manufacturers and operators in many ways, for example obtaining information of the health of equipment, estimating and extend the remaining service life of equipment, increase plant availability and planning of maintenance schedule [1] [2] [3]. There a number of condition monitoring techniques for transformers such as Dissolved Gas Analysis (DGA), Thermal Analysis, Vibration Analysis, Frequency Response Analysis and also the well-known preventive maintenance technique, PD Analysis [4] [5] [6]. Even though the approaches are different, they have similar objectives; which is to obtain information at early stage before a catastrophic failure occurs. This project is primarily concerned with PD analysis.

In a typical environment, PD activity may not only occur from a single source and therefore PD in HV transformer windings may occur from multiple sources simultaneously. An experiment has been designed at the Tony Davies High Voltage Laboratory, University of Southampton to access the methodologies for identification of multiple PD sources within a HV transformer winding. The experiment facilitates the testing of transformer winding under conditions similar to those found in a large power transformer. Previous work [7], in Southampton has developed non-linear based techniques that facilitate identification of the location of a PD source within an interleaved winding. This project is concerned with the feasibility of locating several PD sources simultaneously based only on measurement data from a pair of wideband radio frequency current transformers (RFCTs) positioned at the neutral to earth point and the bushing tap-point to earth. Initial results from a simple experiment have been presented and the developments of an analytical approach is presented.

\section{EXPERIMENTAL ARRANGEMENT}

\section{A. Measurement System}

The experiment consists of a HV transformer winding section, a $60 \mathrm{kV}$ transformer bushing, measurement sensors, PD sources and an oscilloscope as shown in Fig. 1. The HV transformer winding section was used to study PD signal propagation inside a transformer winding. The model of the transformer winding was manufactured by Alstom, a largescale transformer manufacturing company in the UK. The model consists of two sections; an interleaved winding located at the top and the bottom part of the winding section consists of a plain disc winding. The entire winding section is immersed in an oil filled tank filled with oil specification of BS148:1998 class 1, which is discharge-free up to the $30 \mathrm{kV}$ test voltage [7]. In this paper, only PD activity along the interleaved disc type winding has been studied.

The $60 \mathrm{kV}$ transformer bushing is included to be more representative of transformers and account for any attenuation or distortion of higher frequency components of a discharge that are detected via the measurement sensor at the bushing tap point.

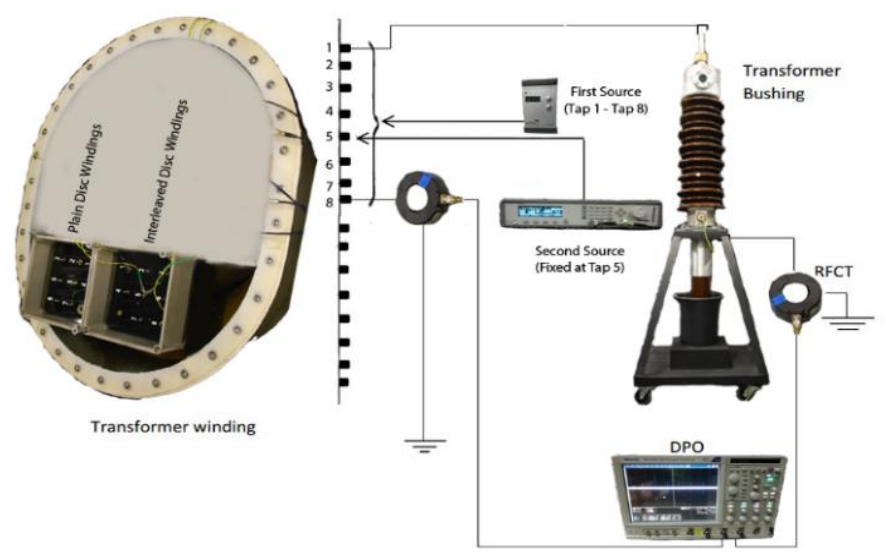

Fig. 1. Experiment Setup

Two RFCTs were used as the measurement sensors to capture the propagated pulses in the winding and were placed at the neutral to earth point and the bushing tap-point to earth. 
The use of an RFCT has been demonstrated to be able to capture high frequency transient signals that are proportional to the time derivative of current in a conductor. Hence the RFCT is an ideal sensor to monitor high frequency discharge currents [8]. In order to increase the sensitivity of each RFCT, both RFCTs were wrapped with three turns of wire conductor. The artificial PD sources that were injected into the winding were generated by two sources and a high performance digital oscilloscope was used in this experiment with a sampling rate set to $100 \mathrm{MSs}^{-1}$ and a bandwidth of $2.5 \mathrm{GHz}$ respectively. The RFCTs used are of the same design and have a comparable frequency response up to $200 \mathrm{MHz}$ and were connected to the oscilloscope for display, analysis and recording of data.

\section{B. Artificial PD Sources}

Two different types of equipment were used to produce different electrical pulses as, "artificial" PD sources in this investigation and they comply with IEC 60270. They were used to inject signals at different locations along the transformer windings. They were an Agilent Pulse Generator and an Omicron PD Calibrator.

An example of pulses produced by the pulse generator is shown in Fig. 2(a). The pulse generator was set to generate the pulses with a rise time $(\Delta \mathrm{t})$, of $11.75 \mathrm{~ns}$, a peak amplitude $(\Delta \mathrm{V})$, of $3.808 \mathrm{~V}$ and a peak pulse frequency $(1 / \Delta \mathrm{t})$ of $85.106 \mathrm{MHz}$. While, the second PD pulse, generated by the PD Calibrator is shown in Fig. 2(b) has a rise time $6.66 \mathrm{~ns}$, a peak amplitude of $1.34 \mathrm{~V}$ and a peak frequency of 150.2 MHz. However, the rise time, peak amplitude and the frequency of the rest of the pulses is not the same because the signals produce by the generator may suffer distortion and attenuation during the experiments.

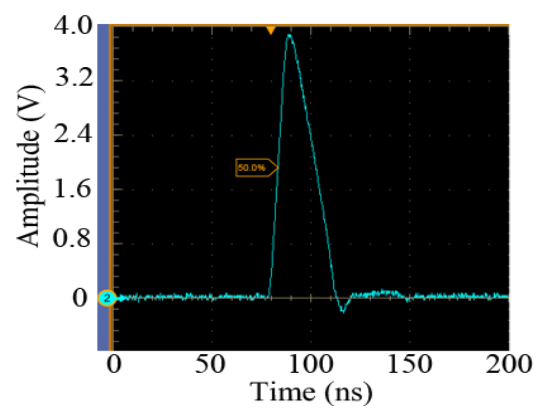

(a) Pulse from Agilent Pulse Generator

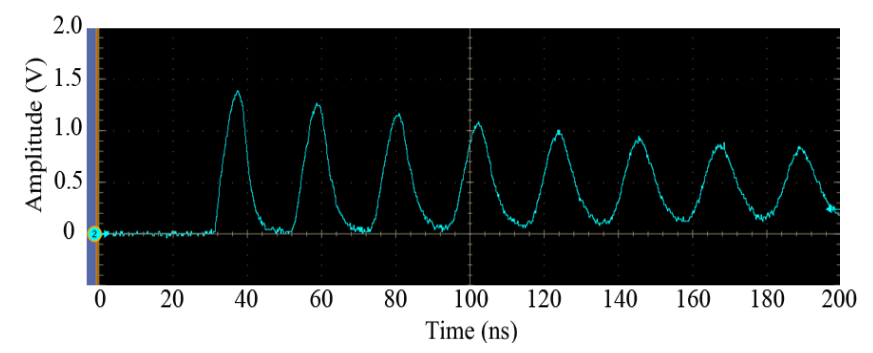

(b) Pulse from Omicron PD Calibrator

Fig. 2. The generated artificial PD pulses

Although in Fig. 2 (b) there are several reflections, the frequency of the pulses are still equivalent to typical internal partial discharge frequency ranges. The artificial "PD-like" pulse sources were used in this study due to their consistency, ease of use and to verify whether the proposed analytical technique is feasible.

\section{SIGNALS PROCESSING}

\section{A. Signal Extraction}

Due to the operation of the pulse generation hardware, a DC offset was produced in the captured signals. Therefore, the DC offset was removed from the raw data to allow further processing. This was completed by subtracting the mean amplitude of the entire cycle from each individual sample. Other challenges with the signals captured by the RFCT is the background noise. It is important to remove the noise in order to extract PD pulses with confidence. In order to remove the noise, before setting the threshold value, it is important to identify the value of background noise via the signal plot of raw data which clearly show the noise level. The threshold value must be set carefully, because if the threshold value is too high, there is possibility of loss of PD pulse information. Otherwise, if the value is too low, noise pulses might be interpreted as PD events. After removing the DC offset and the background noise, the raw signal at both measurement points is assumed to only contain PD pulses of interest.

\section{CLUSTERING AND IDENTIFICATION}

\section{A. Wavelet Analysis}

The original PD pulses were decomposed into nine levels and are presented in the form of ten element vectors. The wavelet transform works like a pair of complementary high-pass (HPF) and low-pass filters (LPF), which divide up of the original signal (S), into a series of approximation coefficients (cA1) and detail coefficients (cD1) at the $1^{\text {st }}$ level. The process is repeated iteratively with each iteration producing another approximation (cA) and detail (cD) coefficients. The first approximation coefficients (cA1) which are decomposed from the original signal (S) will again decompose into the next level, produced a new approximation coefficient (cA2) and a new detail approximation (cD2) at the $2^{\text {nd }}$ level. This process is repeated up to a certain level of decomposition (n-level). Fig. 3 shows the iterative decomposition process of the wavelet transform where $(\mathrm{cA})$ and $(\mathrm{cD})$ represent the approximate and detailed coefficients with the Wavelet filter; LPF and HPF.

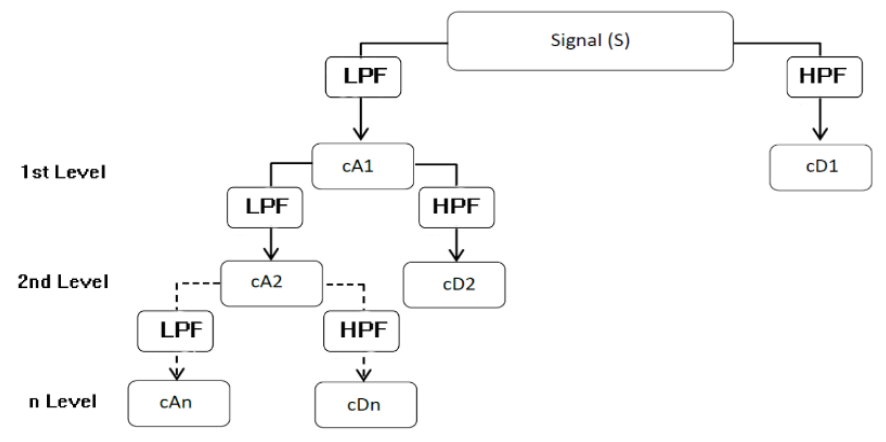

Fig. 3: Iterative decomposition process 
By using the series of approximation coefficients (cA) and detail coefficients $(\mathrm{cD})$ for $n$-levels, the distribution of signal energy in each decomposition level $\left(\mathrm{E}_{\mathrm{D}}\right)$ and final approximation level $\left(\mathrm{E}_{\mathrm{A}}\right)$ can be calculated using (1) and (2) [7] [9]:

$$
\begin{aligned}
& E_{D i}=\frac{\sum_{j=1}^{N c i} C d_{i_{j}}^{2}(t)}{\sum_{i=1}^{n} \sum_{j=1}^{N c i} C d_{i_{j}}^{2}(t)+\sum_{j=1}^{N c n} C a_{n}^{2}(t)} \times 100 \\
& E_{A n}=\frac{\sum_{j=1}^{N c n} C a_{n}^{2}(t)}{\sum_{i=1}^{n} \sum_{j=1}^{N c i} C d_{i_{j}}^{2}(t)+\sum_{j=1}^{N c n} C a_{n}^{2}(t)} \times 100
\end{aligned}
$$

Where $\mathrm{n}, \mathrm{Cd}$ and $\mathrm{Ca}$ are the decomposition level, detail decomposition coefficients and approximate decomposition coefficients. In term of data dimensionality reduction, these energy levels were found to be more effective at representing the PD pulse compared to just using wavelet decomposition coefficients [9].

\section{B. Principle Component Analysis (PCA)}

In order to reduce the number of the element vectors obtained from wavelet analysis to three dimensions, PCA has been applied. The data first is standardised by subtracting the mean value for each dimension to provide zero mean and unity variance. The covariance matrix $\left(\mathrm{C}_{\mathrm{m}}\right)$ can be obtained using the Equation 3 below where the wavelet energy (WE) distribution data is $\mathrm{M}$ and $\mathrm{N}$ is the processed PD pulses:

$$
C_{m}=\frac{M \cdot M^{T}}{N-1}
$$

From the covariance matrix, the eigenvalues $(\lambda)$ and eigenvectors ( $v)$ are obtained and the eigenvalues are arranged in descending order. The matrix $\left(\mathrm{C}_{\mathrm{m}}\right)$ is transposed into a projection, $\mathrm{P}_{\mathrm{i}}$ using the eigenvectors (v) such that in (4):

$$
P_{i}=\mathrm{v}^{T} \times M
$$

Therefore, each row of the projection, $\mathrm{P}_{\mathrm{i}}$ represents principal components, with decreasing significance. The new representation of the data in 3-D feature space can be plotted using the three lower order of the principal components [9] [7] [10].

\section{Density-Based Spatial Clustering of Applications with} Noise (DBSCAN)

DBSCAN was applied to the data in 3-D space to groups the data into specific classes or clusters accordingly based on the concept of density. The DBSCAN algorithm uses three important parameters which are Epsilon $(\varepsilon)$, minimum number of points (n-minimum) and the volume (v). The basic ideas of DBSCAN involve a number of definitions. The first step of DBSCAN is that the density represents the number of points $(\mathrm{n})$, surrounding a certain point of data $\left(\mathrm{p}_{\mathrm{n}}\right)$, within a small volume $(\mathrm{v})$. The volume can be assumed as a hyper-sphere of radius $\varepsilon$ centered at $\mathrm{p}_{\mathrm{n}}$. By using $\mathrm{n}$-minimum, the threshold density can be specified to make the volume $\mathrm{v}$, significantly dense. The point $\mathrm{p}_{\mathrm{n}}$ can be defined as either a dense point, known as a core point, or a non-dense point (border point or non-core point). The rules used to define the method are [9] [10]:

- If a number of points ( $\mathrm{n}$ ) contains at least a minimum number ( $\mathrm{n} \geq \mathrm{n}$-minimum) of points within the radius $\varepsilon$, centred at $\mathrm{p}_{\mathrm{n}}$ and are included in the volume $\mathrm{v}$, the point $\mathrm{p}_{\mathrm{n}}$ is called a core point.

- $\quad \mathrm{p}_{\mathrm{n}}$ is called a border point if a number of points ( $\mathrm{n}$ ) contains at less than minimum number $(n<n-$ minimum) of points within the radius $\varepsilon$, centred at $\mathrm{p}_{\mathrm{n}}$ and are included in the volume $\mathrm{v}$.

- If point $\mathrm{q}$ is included in $\mathrm{v}$, within the radius $\varepsilon$ and $\mathrm{p}_{\mathrm{n}}$ is a core point, point $\mathrm{q}$ is called directly-density reachable (DDR) from point $\mathrm{p}_{\mathrm{n}}$.

- A point $r$ is called density reachable (DR) from $p_{n}$ within volume $\mathrm{v}$ with respect to the radius $\varepsilon$, if there is a chain of objects $p_{1}, p_{2} \ldots \ldots p_{k}$, where $p_{1}=p_{n}, p_{k}=r$ such that, $\mathrm{p}_{\mathrm{i}+1}$ is DDR from $\mathrm{p}_{\mathrm{i}}$.

- A point $\mathrm{s}$ is called density-connected (DC) to $\mathrm{p}_{\mathrm{n}}$ with respect to radius $\varepsilon$ if there is a point $t$ such that both points, $s$ and $\mathrm{p}_{\mathrm{n}}$ are density reachable from point $\mathrm{t}$ with respect to the radius $\varepsilon$.

- Any point which not belong to any group of point $\left(\mathrm{p}_{\mathrm{n}}\right.$, $\mathrm{q}, \mathrm{r}$ or $\mathrm{s}$ ), it is considered as a noise points.

DBSCAN then check the condition of point $\mathrm{p}_{\mathrm{n}}$ for any given $\varepsilon$ and $n$-minimum value. If the point $\mathrm{p}_{\mathrm{n}}$ is a core point, it selects the DR and DDR points and expands the cluster by merging neighbouring dense regions together. If $\mathrm{p}_{\mathrm{n}}$ is a border point then no points are DR from $p_{n}$ and DBSCAN visits the next point of the database. The point $p_{n}$ initially can be selected randomly or resorting to the maximum or minimum weight of a selected function. Then, once the border of the first cluster is identified, DBSCAN will select another point $\mathrm{p}_{\mathrm{n}}$ in the 3-D space which does not belong to a previous cluster and this process is a repetitive process and will terminates when no new point can be detected to add to any previous formed clusters [9] [10].

\section{RESULTS}

Fig. 4 shows the 3D plot obtained from PCA and DBSCAN for multiple PD sources injected simultaneously. There are four classes which represent two different sources at two measurement points, placed at the neutral to earth point and the bushing tap-point to earth. From DBSCAN analysis output, it was known that Class 1 and Class 2 are produced from the pulses obtained at bushing tap-point to earth while Class 3 and Class 4 are produced from the pulses obtained at neutral to earth point. The question arises, which classes belong to the first source and which classes belong to the second source?

Fig. 5 and Fig. 6 show the plots for frequency for each pulse at terminal 1 and terminal 5. By inspection, there are clear similarities in terms of the frequency content of Class 1 and Class 3 and Class 2 is most similar to Class 4 . 


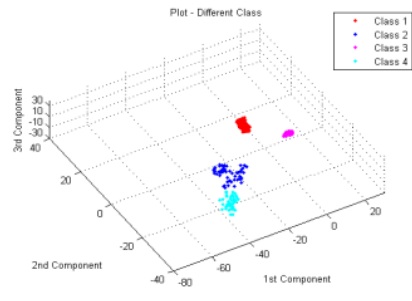

(a) Terminal 1

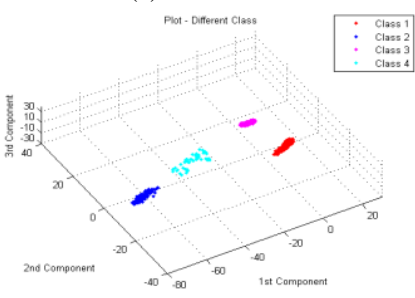

(e) Terminal 5

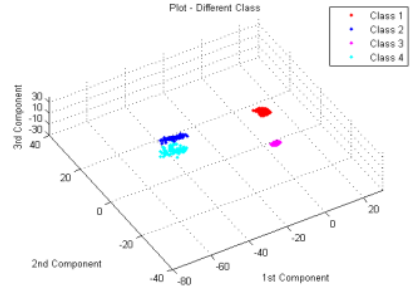

(b) Terminal 3

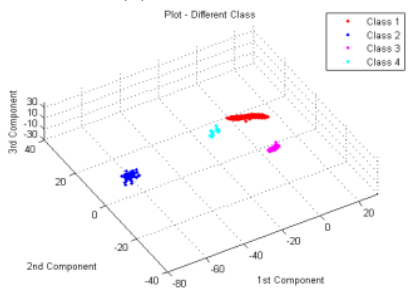

(f) Terminal 7
Fig. 4. 3D plot obtained from PCA and DBSCAN for multiple PD sources injected simultaneously

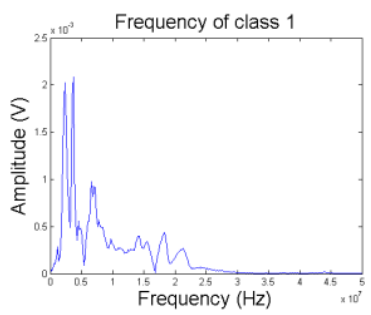

(a) Class 1

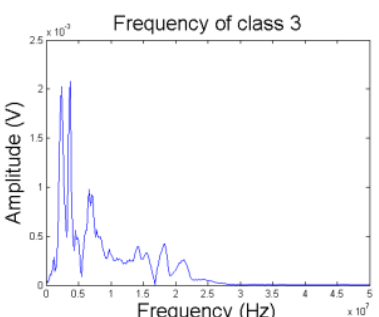

(c) Class 3

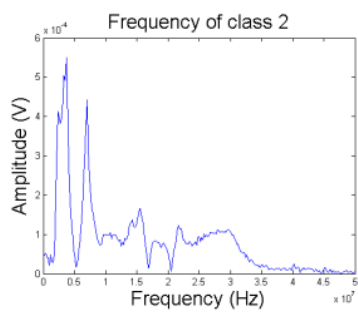

(b) Class 2

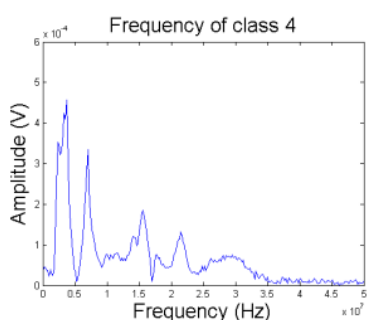

(d) Class 4
Fig. 5. The pulse frequency of each class at terminal 1

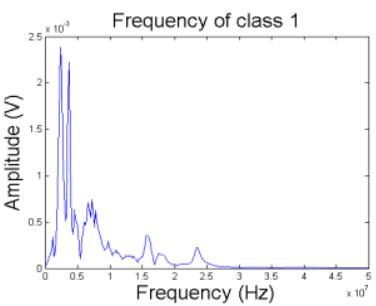

(c) Class 1

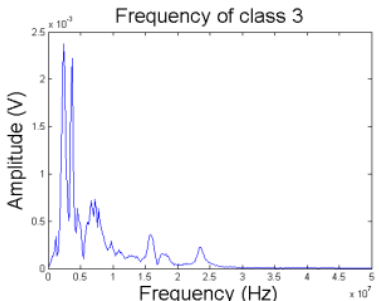

(c) Class 3

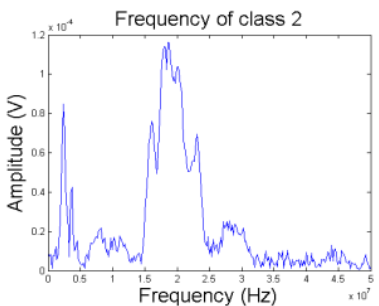

(d) Class 2

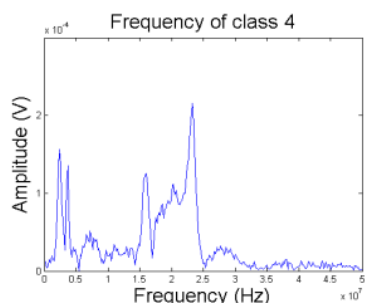

(d) Class 4
Fig. 7. The pulse frequency of each class at terminal 5

\section{CONCLUSION}

The use of clustering along with signal frequency analysis may allow the identification of two artificial "PD-like" pulses within a large transformer. Although the distinct classification of individual source types is not currently possible, the relative variation in frequency distribution is such that multiple sources can be isolated from each other for further analysis. This initial study was a feasibility exercise to see if there would be significant separation between clustered data from multiple sources and whether if may be possible to use information within the measured pulses to determine which pulses are associated with a particular source. The results obtained are very promising and further work will concentrate on refining the analytical technique whilst conducting experiments more closely matched to conditions in the field.

\section{REFERENCES}

[1] E. Gockenbach and H. Borsi, "Condition monitoring and diagnosis of power transformers," International Symposium on Electrical Insulating Materials (ISEIM), pp. 16 - 19, 2008.

[2] D. Harris and M. Saravolac, "Condition monitoring in power transformers," IEE Colloquium on Condition Monitoring of Large Machines and Power Transformers (Digest No: 1997/086), pp. 7/1 7/3, 1997.

[3] A. White, "A transformer manufacturer's perspective of condition monitoring systems," HV Measurements, Condition IEE Colloquium on Monitoring and Associated Database Handling Strategies (Ref. No. 1998/448), pp. 4/1 - 4/4, 1998.

[4] A. E. B. Abu-Elanien and M. M. A. Salama, "Survey on the Transformer Condition," Large Engineering Systems Conference on Power Engineering, pp. 187 - 191, 2007.

[5] J. S. Y. Singh and R. Jarial, "Condition Monitoring of Power Transformers - Bibliography Survey," Electrical Insulation Magazine, IEEE, vol. 24, no. 3, pp. 11 - 25, 2008.

[6] T. Saha, "Review of modern diagnostic techniques for assessing insulation condition in aged transformers," IEEE Transactions on Dielectrics and Electrical Insulation, vol. 10, no. 5, pp. 903 - 917 2003.

[7] M. S. A. Rahman, "Identification of Partial Discharge Sources and Their Location within High Voltage Transformer Windings," Ph.D. thesis, School Electronics and Computer Science, University of Southampton, 2014.

[8] L. Hao, P. Lewin and S. Swingler, "Identification of multiple partial discharge sources," International Conference on Condition Monitoring and Diagnosis, pp. 118 - 121, 2008.

[9] L. Hao, P. Lewin, J. Hunter, D. Swaffield, A. Contin, C. Walton and M Michel, "Discrimination of multiple PD sources using wavelet decomposition and principal component analysis," IEEE Transactions on Dielectrics and Electrical Insulation, vol. 18, no. 5, pp. 1702 - 1711, October 2011.

[10] J. A. Hunter, "An investigation into partial discharge," Ph.D. thesis, School Electronics and Computer Science, University of Southampton, 2013. 